\title{
Rescaled range and transition matrix analysis of DNA sequences
}

\author{
$\mathrm{Zu}-\mathrm{Guo} \mathrm{Yu}^{1,2,3}$ and Guo-Yi Chen ${ }^{2}$ \\ ${ }^{1}$ Department of Mathematics, Xiangtan University, Hunan 411105, P.R. China* \\ ${ }^{2}$ Institute of Theoretical Physics, Chinese Academy of Sciences, \\ P.O. Box 2735, Beijing 100080, P. R. China. \\ ${ }^{3}$ CCAST( World Laboratory), P.O. Box 8370, Beijing 100080, P.R. China.
}

\begin{abstract}
In this paper we treat some fractal and statistical features of the DNA sequences. First, a fractal record model of DNA sequence is proposed by mapping DNA sequences to integer sequences, followed by $R / S$ analysis of the model and computation of the Hurst exponents. Second, we consider transition between the four kinds of bases within DNA. The transition matrix analysis of DNA sequences shows that some measures of complexity based on transition proportion matrix are of interest. The main results are:

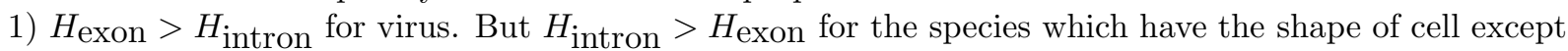
for drosophila. 2) For Virus, E. coli, yeast, drosophila, mouse and human, measures $\mathcal{H}$ of transition proportion matrix of exon is larger than that of intron, and measures $\lambda, \mathcal{D}, \mathcal{C}, \widetilde{D}$ and $\widetilde{C}$ of transition proportion matrix of intron are larger than that of exon. 3) Regarding the evolution, we find that when the species goes higher in grade, the measures $\mathcal{D}, \mathcal{C}, \widetilde{D}$ and $\widetilde{C}$ of exon become larger, the measure $\mathcal{H}$ of exon becomes lesser except for yeast. Hence for species of higher grade, the transition rate among the four kinds of bases goes further from the equilibrium.
\end{abstract}

Key words: DNA sequence, functional region, $R / S$ analysis, transition proportion matrix, measure of complexity.

PACS numbers: $87.10+\mathrm{e}$

\section{Introduction}

In the past decade or so there has been a ground swell of interest in unraveling the mysteries of DNA. In order to distinguish coding regions from non-coding ones, many approaches have been proposed. First, investigation into nucleotide correlation is of special importance. In recent years many authors have discussed the correlation properties of nucleotides in DNA sequences ${ }^{[1-9]}$. C.K. Peng et a ${ }^{[4]}$, using the one-dimensional DNA walk model found that there exists long-range correlation in non-coding regions but not in coding regions. Second, the linguistic approach. DNA sequence can be regarded, at a number of levels, as analogous to mechanisms of processing other kind of languages, such as natural languages and computer languages ${ }^{[10]}$. R.N. Mantegna et al also studied the linguistic feature of non-coding DNA sequences ${ }^{[11]}$. Third, the nonlinear scaling method, such as complexity ${ }^{[12]}$ and fractal analysis ${ }^{[13-17]}$. Recently, we investigated the correlation

* This is the corresponding address of the first author, Email: yuzg@itp.ac.cn 
dimension and Kolmogorov entropy of DNA sequences using time series model ${ }^{[18]}$. Our goal is to search for a good measure of complexity which can be used to clearly distinguish different functional regions of DNA sequences and to describe the evolution of species.

In this paper, we first map DNA sequence to sequence of integer numbers, and treat it like a fractal record in time, then apply $R / S$ analysis to calculate its Hurst exponent. Second. We analyze DNA sequences with the transition matrix method and calculate some measures of complexity based on their transition proportion matrices.

\section{$2 \quad R / S$ analysis}

A DNA sequence may also be regarded as a sequence over the alphabet $\{A, C, G, T\}$, which represents the set of the four bases from which DNA is assembled, namely adenine, cytosine, guanine and thymine. For any DNA sequence $s=s_{1} s_{2} \cdots s_{N}$, we define a map $f: s \mapsto x=\left\{x_{1}, x_{2}, \cdots, x_{N}\right\}$, where for any $1 \leq k \leq N$,

$$
x_{k}= \begin{cases}-2, & \text { if } s_{k}=A \\ -1, & \text { if } s_{k}=C, \\ 1, & \text { if } s_{k}=G \\ 2, & \text { if } s_{k}=T\end{cases}
$$

According to the definition of $f$, the four bases $\{A, C, G, T\}$ are mapped to four distinct value. One can also use $\{-2,-1,1,2\}$ to replace $\{A, G, C, T\}$ or other orders of $A, G, C, T$. our main aim is distinguish $A$ and $G$ from purine, $C$ and $T$ from pyrimidine. We expect it to reveal more information than one dimensional DNA walk ${ }^{[4]}$.

Remark: William Y. C. Chen and J. D. Louck ${ }^{[19]}$ also use the $\{-2,-1,1,2\}$ alphabet for the DNA sequence, instead of $\{A, C, G, T\}$.

Thus we obtain a number sequence $x=\left\{x_{k}\right\}_{k=1}^{N}$, where $x_{k} \in\{-2,-1,1,2\}$. This sequence can be treated as a fractal records in time. To study such sequences, Hurst ${ }^{[20]}$ invented a new statistical method - the rescaled range analysis $\left(R / S\right.$ analysis), then B. B. Mandelbrot ${ }^{[21]}$ and J. Feder ${ }^{[22]}$ introduced $R / S$ analysis of fractal records in time into fractal analysis. For any fractal records in time $x=\left\{x_{k}\right\}_{k=1}^{N}$ and any $2 \leq n \leq N$, one can define

$$
\begin{gathered}
<x>_{n}=\frac{1}{n} \sum_{i=1}^{n} x_{i} \\
X(i, n)=\sum_{u=1}^{i}\left[x_{u}-<x>_{n}\right] \\
R(n)=\max _{1 \leq i \leq n} X(i, n)-\min _{1 \leq i \leq n} X(i, n) \\
S(n)=\left[\frac{1}{n} \sum_{i=1}^{n}\left(x_{i}-<x>_{n}\right)^{2}\right]^{1 / 2} .
\end{gathered}
$$

Hurst found that

$$
R(n) / S(n) \sim\left(\frac{n}{2}\right)^{H}
$$




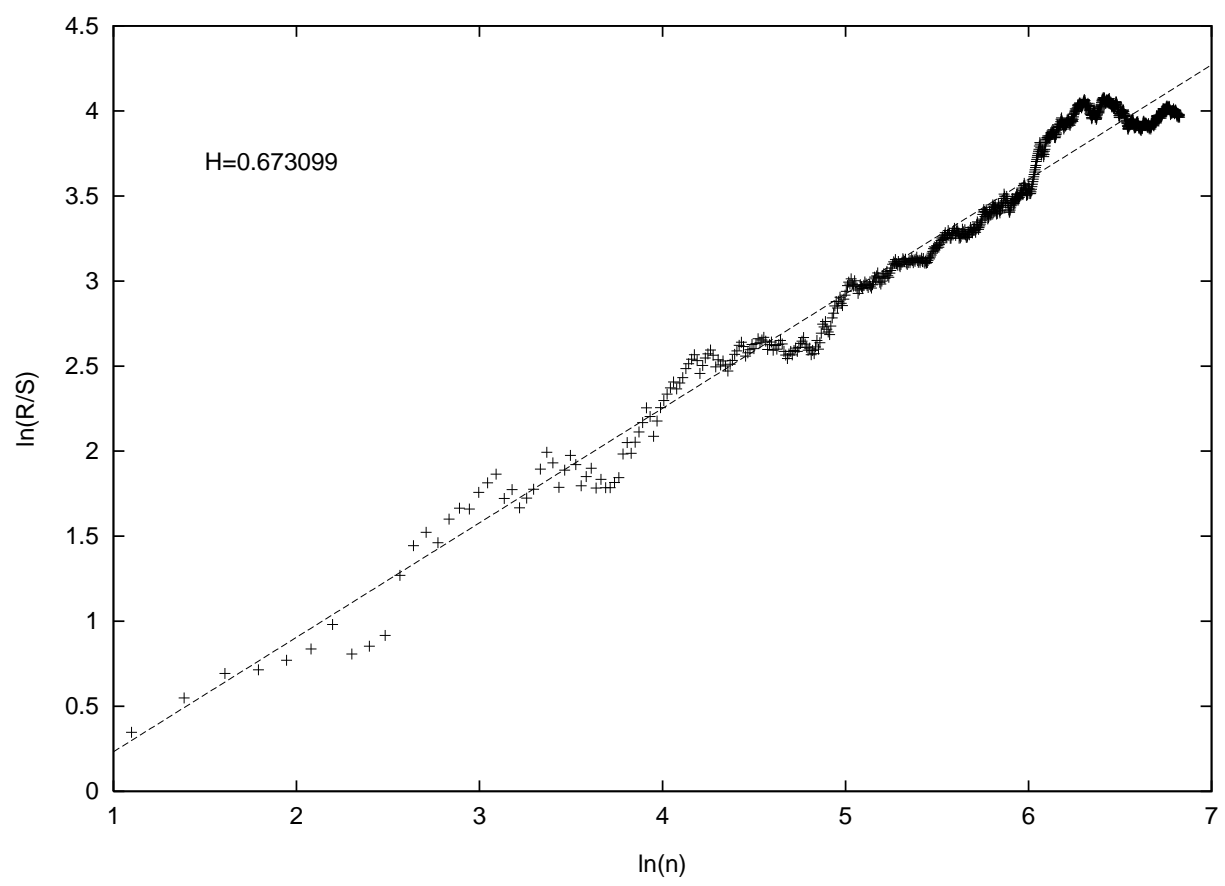

Figure 1: An example of $R / S$ analysis of DNA sequence

$H$ is called Hurst exponent.

As $n$ changes from 2 to $N$, we obtain $N-1$ points in $\ln (n)$ v.s. $\ln (R(n) / S(n))$ plane. Then we can calculate Hurst exponent $H$ of DNA sequence $s$ using the least-square linear fit. As an example, we plot the graph of $R / S$ analysis of an exon segment $s$ of mouse' DNA sequence (bp 1730- bp 2650 of the record with Accession AF033620 in Genbank) in Figure 1.

The Hurst exponent is usually used as a measure of complexity. From Page 149 of Ref.[22], the trajectory of the record is a curve with a fractal dimension $D=2-H$. Hence a smaller $H$ means a more complex system. When applied to fractional Brownian motion, if $H>1 / 2$, the system is said to be persistent, which means that if for a given time period $t$, the motion is along one direction, then in the succeeding $t$ time, it's more likely that the motion will follow the same direction. While for system with $H<1 / 2$, the opposite holds, that is, antipersistent. But when $H=1 / 2$, the system is Brown motion, and is random.

We randomly choose 17 exons and 34 introns from Virus' genome; 8 exons and 9 introns from E. coli's; 22 exons and 22 introns from yeast's; 30 exons and 24 introns from drosophila's; 37 exons and 31 introns from mouse's; 78 exons and 27 introns from Human's( all data from Genbank). The Hurst exponent $H$ s are calculated for each sequence and averaged according to both species category and function, their relative standard deviations are also calculated. We list the results in Table 1 (we briefly write "relative standard deviation" as "RSD" in the following tables).

\section{Transition Matrix analysis}

Readers can see the concept of Transition Matrix of a data sequence in the book of J.C.Davis ${ }^{[23]}$. Here we use this method to study DNA sequences, mainly on the nature of transitions from one kind of base to 
Table 1: Average and relative standard deviation of $H$

\begin{tabular}{|c|c|c|c|c|c|c|c|}
\hline & & virus & E. coli & yeast & drosophila & mouse & human \\
\hline \multirow{2}{*}{ Average } & exon & 0.6017 & 0.5991 & 0.6117 & 0.6135 & 0.5746 & 0.5967 \\
\cline { 2 - 8 } & intron & 0.5536 & 0.6482 & 0.6268 & 0.6003 & 0.6017 & 0.6000 \\
\hline \multirow{2}{*}{ RSD } & exon & 0.1510 & 0.0790 & 0.1442 & 0.1653 & 0.1446 & 0.1471 \\
\cline { 2 - 8 } & intron & 0.2114 & 0.1265 & 0.1558 & 0.1629 & 0.1795 & 0.1526 \\
\hline
\end{tabular}

another, which presents useful information of the sequence.

For a given DNA sequence $s=s_{1} s_{2} \cdots s_{N}$, we can construct a $4 \times 4$ matrix $\mathcal{A}=\left(t_{i j}\right)$, where $t_{i j}$ means the number of times a given kind of base being succeeded by another in the sequence. $\mathcal{A}$ is called the transition frequency matrix of $s$, which is a concise way of expressing the incidence of one kind of base following another. For example, for $s=A T A G C G C A T G T A C G C G T A G A T C A T G C T A G C A$, the transition frequency matrix is shown below:

To

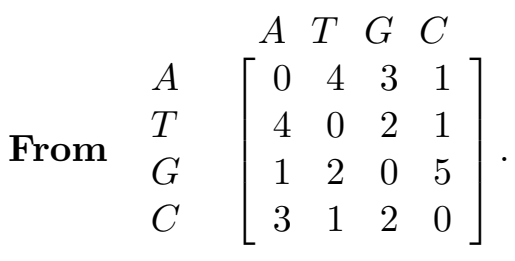

The tendency for one kind of bases to succeed another can be emphasized by converting the frequency matrix to decimal fractions or percentages. Therefore, we can construct a matrix $\mathcal{P}=\left(P_{i j}\right)$ by dividing each element by the grand total of all entries in $\mathcal{A}$. Such a matrix represents the relative frequency of all the possible types of transitions, and is called the transition proportion matrix of $s$. For the above example, the transition proportion matrix is:

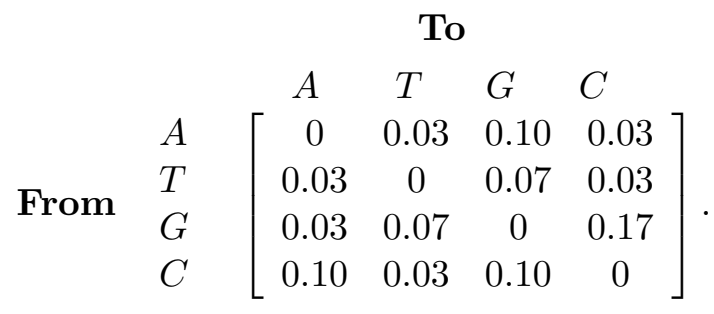

First, We calculate the maximum real eigenvalue $\lambda$ of the transition proportion matrix $\mathcal{P}$ of the DNA sequence. It is natural that such a parameter is relevant to the system's complexity.

Second, Since $\sum_{i, j=1}^{4} P_{i j}=1,0 \leq P_{i j} \leq 1$, we can view $P_{i j}$ as the probability of one kind of base to succeed another. If we denote $\#\left\{P_{i j}: P_{i j} \neq 0\right\}=M$ be the number of probabilities which is not zero, and rewrite $\left\{P_{i j}: P_{i j} \neq 0\right\}$ as $\left\{P_{i}\right\}_{i=1}^{M}$. Then Shannon's ${ }^{[24]}$ definition of information entropy applies

$$
\mathcal{H}=-\sum_{i=1}^{M} P_{i} \ln P_{i} .
$$


Table 2: Average of the maximum real eigenvalue $\lambda$

\begin{tabular}{|c|c|c|c|c|c|c|}
\hline & virus & E. coli & yeast & drosophila & mouse & human \\
\hline exon & 0.2564 & 0.2616 & 0.2663 & 0.2648 & 0.2596 & 0.2711 \\
\hline intron & 0.2913 & 0.28835 & 0.2980 & 0.2839 & 0.2752 & 0.2720 \\
\hline
\end{tabular}

Table 3: Average and relative standard deviation of information entropy $\mathcal{H}$

\begin{tabular}{|c|c|c|c|c|c|c|c|}
\hline & & virus & E. coli & yeast & drosophila & mouse & human \\
\hline Average & exon & 2.6646 & 2.6636 & 2.6282 & 2.6620 & 2.6471 & 2.5954 \\
\cline { 2 - 8 } & intron & 2.5566 & 2.5513 & 2.5241 & 2.5840 & 2.5834 & 2.5884 \\
\hline \multirow{2}{*}{ RSD } & exon & 0.0352 & 0.0212 & 0.0248 & 0.0258 & 0.0215 & 0.0311 \\
\cline { 2 - 8 } & intron & 0.0770 & 0.0268 & 0.0401 & 0.0398 & 0.0372 & 0.0339 \\
\hline
\end{tabular}

When $P_{i}=1 / M, i=1,2, \cdots, M$, i.e. the case of equilibrium state, the function $\mathcal{H}\left(P_{1}, \cdots, P_{M}\right)$ reaches its maximum value. When $P_{i}=1$ for some $i$ and $P_{j}=0$ for $j \neq i$, we have $\mathcal{H}\left(P_{1}, \cdots, P_{M}\right)=0$.

There is also a definition of disequilibrium $\mathcal{D}{ }^{[25]}$, used as a measure of "complexity" in $M$-system.

$$
\mathcal{D}=\sum_{i=1}^{M}\left(P_{i}-\frac{1}{M}\right)^{2} .
$$

When $P_{i}=1 / M, i=1,2, \cdots, M$, i.e. the case of equilibrium state, the function $\mathcal{D}=0$. When $P_{i}=1$ for some $i$ and $P_{j}=0$ for $j \neq i, \mathcal{D}$ gets its maximum value.

R. Lope-Ruiz et al ${ }^{[26]}$ proposed another statistical measure of complexity $\mathcal{C}$, which is defined as

$$
\mathcal{C}=\mathcal{H} \times \mathcal{D} .
$$

Now $\mathcal{C}=0$ for both the equilibrium state and the case of $P_{i}=1$ for some $i$ and $P_{j}=0$ for $j \neq i$.

We also define two more measures of complexity as follows:

$$
\begin{gathered}
\widetilde{D}=\left[\mathcal{D} /\left(\frac{1}{M} \sum_{i=1}^{M} P_{i}^{2}\right)\right]^{1 / 2} \\
\widetilde{C}=\mathcal{H} \times \widetilde{D} .
\end{gathered}
$$

$\widetilde{D}$ means the relative disequilibrium. They are inspired by $\mathcal{D}$ and $\mathcal{C}$, but exhibit better behavior in the computation.

For DNA sequences chosen in the previous section, The measures $\lambda, \mathcal{H}, \mathcal{D}, \mathcal{C}, \widetilde{D}$ and $\widetilde{C}$ of complexity are calculated for each sequence and averaged according to both biological category of species and the function. In addition, the relative standard deviations of $\mathcal{H}, \mathcal{D}, \mathcal{C}, \widetilde{D}$ and $\widetilde{C}$ are also calculated. The results are listed in Table 2-7. 
Table 4: Average and relative standard deviation of $\mathcal{D}$

\begin{tabular}{|c|c|c|c|c|c|c|c|}
\hline & & virus & E. coli & yeast & drosophila & mouse & human \\
\hline \multirow{2}{*}{ Average } & exon & 0.0121 & 0.0123 & 0.0172 & 0.0137 & 0.0146 & 0.0211 \\
\cline { 2 - 8 } & intron & 0.0317 & 0.0275 & 0.0331 & 0.0250 & 0.0242 & 0.0234 \\
\hline \multirow{2}{*}{ RSD } & exon & 0.5986 & 0.4197 & 0.4086 & 0.5277 & 0.4082 & 0.4260 \\
\cline { 2 - 8 } & intron & 0.7604 & 0.2823 & 0.4501 & 0.5147 & 0.5236 & 0.5005 \\
\hline
\end{tabular}

Table 5: Average and relative standard deviation of $\mathcal{C}$

\begin{tabular}{|c|c|c|c|c|c|c|c|}
\hline & & virus & E. coli & yeast & drosophila & mouse & human \\
\hline \multirow{2}{*}{ Average } & exon & 0.0313 & 0.0325 & 0.0448 & 0.0360 & 0.0382 & 0.0540 \\
\cline { 2 - 8 } & intron & 0.0739 & 0.0697 & 0.0820 & 0.0631 & 0.0612 & 0.0595 \\
\hline \multirow{2}{*}{ RSD } & exon & 0.5614 & 0.4038 & 0.3912 & 0.5078 & 0.3846 & 0.3862 \\
\cline { 2 - 8 } & intron & 0.7203 & 0.2660 & 0.4102 & 0.4915 & 0.4883 & 0.4629 \\
\hline
\end{tabular}

Table 6: Average and relative standard deviation of $\widetilde{D}$

\begin{tabular}{|c|c|c|c|c|c|c|c|}
\hline & & virus & E. coli & yeast & drosophila & mouse & human \\
\hline \multirow{2}{*}{ Average } & exon & 0.3767 & 0.3925 & 0.4492 & 0.4008 & 0.4226 & 0.4871 \\
\cline { 2 - 8 } & intron & 0.4852 & 0.5434 & 0.5679 & 0.4999 & 0.4996 & 0.5000 \\
\hline \multirow{2}{*}{ RSD } & exon & 0.2545 & 0.1919 & 0.1832 & 0.2434 & 0.1654 & 0.1579 \\
\cline { 2 - 8 } & intron & 0.3416 & 0.1210 & 0.1469 & 0.2428 & 0.2105 & 0.1775 \\
\hline
\end{tabular}

Table 7: Average and relative standard deviation of $\widetilde{C}$

\begin{tabular}{|c|c|c|c|c|c|c|c|}
\hline & & virus & E. coli & yeast & drosophila & mouse & human \\
\hline \multirow{2}{*}{ Average } & exon & 0.9949 & 1.0413 & 1.1754 & 1.0603 & 1.1149 & 1.2584 \\
\cline { 2 - 8 } & intron & 1.2070 & 1.3821 & 1.4254 & 1.2794 & 1.2809 & 1.2865 \\
\hline \multirow{2}{*}{ RSD } & exon & 0.2160 & 0.1721 & 0.1613 & 0.2190 & 0.1439 & 0.1286 \\
\cline { 2 - 8 } & intron & 0.2722 & 0.1002 & 0.1105 & 0.2122 & 0.1774 & 0.1435 \\
\hline
\end{tabular}




\section{Conclusions}

Virus is species which has not the shape of cell. E. coli belongs to prokaryote and has the shape of cell. Yeast, drosophila, mouse and human belong to eukaryote and also have the shape of cell. From the point of view of evolution, virus has lower grade than E. coli; E. coli has lower grade than that of yeast which has lower grade than that of drosophila; drosophila has lower grade than that of mouse which has lower grade than that of human. We use $H_{\text {exon }}$ to denote the Hurst exponent of exon, and similarly for other measures of complexity and functional regions of DNA.

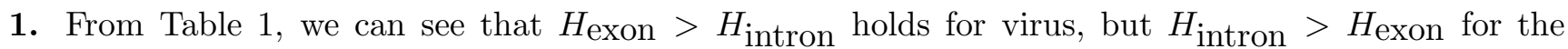
species which have the shape of cell except for drosophila. The latter means that exons are more complex than introns. This result coincides with the conclusion of Ref.[12, 14,18]. From Table 1 we also find that the Hurst exponent of DNA sequence is generally larger than $\frac{1}{2}$. This means that when we use fractional Brownian motion model to describe DNA sequences, we can say it is a persistent system. In particular, we can see $H_{\text {exons }}$ are different from $1 / 2$ explicitly. It indicates that coding regions of DNA is far from random. This is different from the result of Ref.[4] and coincides with the results of Ref.[14]. But we can not find any trend that coincides with the evolution in Table 1.

When we consider the transition of bases in DNA sequence, then

2. For Virus, E. coli, yeast, drosophila, mouse and human, from Table 3, we can conclude that measure $\mathcal{H}$ of transition proportion matrix of exon is larger than that of intron, and measures $\lambda, \mathcal{D}, \mathcal{C}, \widetilde{D}$ and $\widetilde{C}$ of transition proportion matrix of intron are larger than that of exon.

3. Regarding the evolution, we find that as the grade goes higher, measures $\mathcal{D}, \mathcal{C}, \widetilde{D}$ and $\widetilde{C}$ of exon become larger, the measure $\mathcal{H}$ of exon becomes lesser except for yeast. Hence for exon of species of higher grade, the transition statistics of the four kinds of bases goes further from equilibrium.

From the above tables, one can find the information entropy $\mathcal{H}$ has the less relative standard deviation than other measures of complexity.

4. From the previous discussions, we find that measure $\mathcal{H}$ is a good measure of complexity which can be used to clearly distinguish different functional regions of DNA sequences and to describe the evolution of species.

\section{ACKNOWLEDGMENTS}

The authors would like to express their gratitude toward Prof. Bai-lin Hao for introduction into this field, useful discussions and encouragement. And to Prof. Wei-Mou Zheng, Dr. Zuo-Bing Wu and Yang Zhang for many helpful discussions. This project was partially supported by China postdoctoral Science Fundation No. 98B632.

\section{References}

[1] W. Li and K. Kaneko, Europhys. Lett. 17 (1992) 655.

[2] A. Grosberg, Y. Rabin, S. Havlin, and A. Neer, Europhys. Lett. 23 (1993) 373.

[3] (a) R. Voss, Phys. Rev. Lett. 68 (1992) 3805; (b) Fractals 2 (1994) 1.

[4] C.K. Peng, S. Buldyrev, A.L.Goldberg, S. Havlin, F. Sciortino, M. Simons, and H.E. Stanley, Nature 356 (1992) 168.

[5] H.E. Stanley, S.V. Buldyrev, A.L. Goldberg, Z.D. Goldberg, S. Havlin, R.N. Mantegna, S.M. Ossadnik, C.K. Peng, and M. Simons, Physica A 205 (1994) 214. 
[6] H.Herzel, W. Ebeling, and A.O. Schmitt, Phys. Rev. E 50 (1994) 5061.

[7] P. Allegrini, M. Barbi, P. Grigolini, and B.J. West, Phys. Rev. E 52 (1995) 5281.

[8] S.V. Buldyrev, N.V. Dokholyan, A.L. Goldberger, S. Havlin, C.-K. Peng, H.E. Stanley and G.M. Visvanathan, Physica A 249 (1998) 430-438.

[9] Liaofu Luo, Weijiang Lee, Lijun Jia, Fengmin Ji and Lu Tsai, Phys. Rev. E 58(1) (1998) 861-871.

[10] D.B. Searls, CABIOS 13 (1997) 333-344.

[11] R.N. Mantegna, S.V. Buldgrev, A.L. Goldberger, S. Havlin, C.-K. Peng, M. Simons and H.E. Stanley, Phys. Rev. Lett. 73(23) (1994) 3169-3172.

[12] Ruqun Shen, Rensheng Chen, Lunjiang Lin, Jian Sun, Yi Xiao, and Jun Xu, Chinese Science Bulletin (in Chinese) 38 (1993) 1995-1997.

[13] L.F. Luo and L. Tsai, Chin. Phys. Lett. 5 (1988) 421-424.

[14] Liaofu Luo and Lu Tsai, DNA walk and fractal analysis of nucleotide sequence, to appear in Phys. Rev. E.

[15] C.L. Berthelsen, J.A. Glazier and M.H. Skolnick, Phys. Rev. A 45 (1992) 8902.

[16] C.L. Berthelsen, J.A. Glazier and S. Raghavachari, Phys. Rev. E 49 (1994) 1860.

[17] P. Bernaola-Galvan, R. Roman-Roldan and J. L. Oliver, Phys. Rev. E 53 (1996) 5181.

[18] Zu-Guo Yu, Correlation dimension and Kolmogorov entropy of DNA sequence, submitted to Chinese Science Bulletin.

[19] William Y. C. Chen and James D. Louck "Necklaces, MSS Sequences, DNA Sequences" Adv. in Appl. Math. 18(1) (1997) 18-32.

[20] H.E. Hurst, Long-term storage capacity of reservoirs, Trans. Amer. Soc. Civ. Eng. 116 (1951) 770-808.

[21] B.B. Mandelbrot, The Fractal Geometry of Nature, W. H. Freeman, New York, 1982.

[22] J. Feder, Fractals, Plenum Press, New York, London, 1988.

[23] J.C. Davis, Statistics and Data Analysis in Geology, John \& sons, INC, New York, London, Sydney, Toronto, 1973.

[24] C.E. Shannon and W. Weaver, The Mathematical Theory of Communication, University of Illinois Press, Urbana, IL, 1949.

[25] G. NiE. colis and I. Prigogine, Self-organisation in Nonequilibrium Systems, Wiley, New York, 1977.

[26] R. Lopez-Ruiz, H.L. Mancini, X. Calbet, Phys. Lett. A 209 (1995) 321-326. 